\title{
Corrigendum: Portal vein thrombosis - a primer for the general physician
}

Authors: Mohammad Haris ${ }^{A}$ and Jecko Thachil ${ }^{B}$

Authors: ${ }^{A}$ medical student, University of Manchester, Manchester, UK; ${ }^{B}$ consultant haematologist, Manchester Royal Infirmary, Manchester, UK

Clinical Medicine 2016;17:212-9

Mohammad Haris' name was published with the incorrect spelling. The correct spelling is printed above.

\section{Corrigendum: Drug therapy in anticoagulation: which drug for which patient?}

\author{
Authors: Carolyn M Millar ${ }^{A}$ and Mike A Laffan ${ }^{B}$
}

Authors: A clinical senior lecturer, Imperial College, London, UK and consultant haematologist, Imperial College Healthcare NHS Trust,

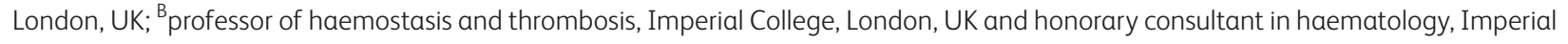
College Healthcare NHS Trust, London, UK

Clinical Medicine 2016;17:233-44

There were errors in the drug doses published in Table 1 and Table 5.

In Table 1, the correct dose of edoxaban should have been listed as $60 \mathrm{mg} \mathrm{od}^{4}$ for NVAF and $60 \mathrm{mg}$ od (following $\geq 5$ days LMWH) ${ }^{4}$ for VTE; the correct dose for rivaroxaban in ACS is $2.5 \mathrm{mg}$ bd, not $2.5 \mathrm{mg}$ od as originally listed.

In Table 5, the correct dose for dabigatran is $110 \mathrm{mg}$ bd and the correct standard dose for rivaroxaban is $20 \mathrm{mg}$ od.

The corrected tables are published below.

There was an omission in Table 2 - the confidence interval for the primary endpoint SSE for edoxaban was $97.5 \%$. 